\title{
Recent Nuclear Astrophysics Data Activities in the U.S.
}

\author{
Michael S. Smith ${ }^{1}$, Daniel W. Bardayan ${ }^{1,2}$, Jeffery C. Blackmon ${ }^{1}$, \\ Edgardo Browne ${ }^{3}$, Richard B. Firestone ${ }^{3}$, Gerald M. Hale ${ }^{4}$, \\ Robert D. Hoffman ${ }^{5}$, Zhanwen Ma ${ }^{1,6}$, Victoria McLane ${ }^{7}$, Eric B. \\ Norman ${ }^{3}$, Nengchuan Shu ${ }^{1,6}$, Donald L. Smith ${ }^{8}$, Laura A. Van \\ Wormer ${ }^{9}, \operatorname{Stan} \mathrm{E}$. Woosley ${ }^{10}$, and Shiu-Chin $\mathrm{Wu}^{3,11}$
}

\author{
${ }^{1}$ Physics Division, Oak Ridge National Laboratory \\ ${ }^{2}$ Yale University \\ ${ }^{3}$ Lawrence Berkeley National Laboratory \\ ${ }^{4}$ Los Alamos National Laboratory \\ ${ }^{5}$ Lawrence Livermore National Laboratory \\ ${ }^{B}$ Chinese Institute for Atomic Energy, Peoples Republic of China \\ ${ }^{7}$ National Nuclear Data Genter, Brookhaven National Laboratory \\ ${ }^{8}$ Argonne National Laboratory \\ ${ }^{9}$ Hiram College \\ ${ }^{10}$ University of California at Santa Cruz \\ ${ }^{11}$ Tsing-Hua University, Republic of China
}

\begin{abstract}
Measurements in nuclear physics laboratories form the empirical foundation for new, realistic, sophisticated theoretical models of a wide variety of astrophysical systems. The predictive power of these models has, in many instances, a strong dependence on the input nuclear data, and more extensive and accurate nuclear data is required for these models than ever before. Progress in astrophysics can be aided by providing scientists with more usable, accurate, and significant amounts of nuclear data in a timely fashion in formats that can be easily incorporated into their models. A number of recent data compilations, evaluations, calculations, and disseminations that address nuclear astrophysics data needs will be described.
\end{abstract}

\section{OVERVIEW}

Nuclear astrophysics research addresses some of the most fundamental questions in nature: What are the origins of the elements that make up our bodies and our world? How did the solar system, the sun, the stars, and the galaxy form, and how do they evolve? Measurements in the nuclear laboratory provide the empirical foundation for the sophisticated theoretical models of these astrophysical

\footnotetext{
The submitted manuscript hes been authored by a contracior of the U.S. ACO5-960R22464. Accordingly, the U.S. Government retains a monexclusive. royalty-free license to publish or reproduce the published form of this contribution, of allow athers to do so. for U.S. Gavernment purposes."
} 
systems. In many cases, however, new nuclear physics measurements are not rapidly disseminated to the research community nor transformed into formats that can be rapidly incorporated into astrophysical models. This situation reduces the impact of new measurements in the astrophysics community that provided their motivation.

Additionally, recent progress in laboratory nuclear physics coupled with the evolution of fast computers has enabled extremely complex astrophysical calculations of increasing realism. For example, calculations coupling the time-dependent synthesis of hundreds of isotopes via thousands of coupling reactions with multidimensional simulations of explosive stellar environments are now carried out. Such new codes require significantly more, and more accurate, nuclear data than ever before to keep pace with the new observational capabilities of instruments such as the Hubble Space Telescope and the Compton Gamma Ray Observatory. Progress toward the solution of many fundamental problems in astrophysics requires the best predictive power of astrophysical models. However, this predictive power has, in many cases, a strong dependence on the input nuclear data. For these reasons, progress in astrophysics can be aided by providing more usable, accurate, and significant amounts of nuclear data in a timely fashion, especially in formats that the astrophysics research community can easily incorporate into their models.

The following gives a brief description of several recent projects that address the nuclear data needs for astrophysics studies. These projects involve data compilations, structure and reaction cross section evaluations, cross section calculations, and data disseminations.

\section{DECAY DATA EVALUATIONS AND WEAK INTERACTION CALCULATIONS FOR NUCLEAR ASTROPHYSICS}

Radioactive decays of long-lived isotopes play an important role in constraining models of stellar explosions and other phenomena in astrophysics. For example, the decay of ${ }^{56-57} \mathrm{Ni}$, and its products ${ }^{56-57} \mathrm{Co}$, power the light curves of all Type I supernovae and the tails on the light curves of most Type II explosions [1]. The abundances of these nuclei and of ${ }^{44} \mathrm{Ti}$ ejected during explosions can be inferred from these light curves and from the gamma rays they emit during their decays. These abundances in turn constrain the conditions deep inside supernova explosions - specifically the mass cut and the degree of neutronization. The lifetime of ${ }^{44} \mathrm{Ti}$ is particularly important and has been used to constrain the explosion mechanism, the nucleosynthesis, and the light curves of supernova explosions such as Cas A $[2,3]$ and SN 1987A [4].

Other radionuclides are also important in astrophysics. For example, recent observations of ${ }^{26} \mathrm{Al}$ decay in the interstellar medium by the Compton Gamma Ray Observatory [5] are used to constrain models of supernovae, novae, and Wolf-Rayet stars $[6,7]$. In many cases, new evaluations of radionuclide decay data are needed to improve astrophysical model predictions. To address this need, evaluations of 
the decays of the isotopes ${ }^{26} \mathrm{AI},{ }^{44} \mathrm{Ti},{ }^{57} \mathrm{Ni}$, and ${ }^{60} \mathrm{Fe}$ have recently been completed [8-10], and evaluations of several additional isotopes are now in progress. The new evaluated half life $(60 \pm 1 \mathrm{y})$ of ${ }^{44} \mathrm{Ti}$ implies, for example, that approximately $10^{-4}$ solar masses of this isotope were ejected in the Cas A supernova explosion. This smaller amount of ${ }^{44} \mathrm{Ti}$ is easier to explain in terms of recent models of supernova nucleosynthesis.

Temperature- and density-dependent weak interaction rates also play a key role in determining both pre-supernova stellar structure, and the nucleosynthesis both before and during the explosion. Theoretical calculations of such rates [11], used for years in astrophysical models, are based upon nuclear physics and data from twenty years ago. A new set of weak interaction rates for fp-shell nuclei, which have improved agreement with $(p, n)$ measurements, have been calculated by Shell Model Monte Carlo techniques [12]. These new rates are now being put into formats compatible with models of stellar explosions, and will be used in the near future to determine their astrophysical implications.

\section{R-MATRIX CALCULATIONS OF REACTION CROSS SECTIONS}

The cross sections of a number of reactions involving light elements (mass $A<$ 16) that are of crucial importance to astrophysics can be calculated via R-matrix techniques. New evaluated cross sections have been obtained for the reactions $\left.{ }^{3} \mathrm{He}(\mathrm{d}, \mathrm{p}){ }^{4} \mathrm{He}[13],{ }^{12} \mathrm{C}(\alpha, \gamma)\right)^{16} \mathrm{O}[14]$, and ${ }^{13} \mathrm{C}(\alpha, \mathrm{n}){ }^{16} \mathrm{O}[15]$ from R-matrix analyses of the ${ }^{5} \mathrm{Li},{ }^{16} \mathrm{O}$, and ${ }^{17} \mathrm{O}$ systems, respectively.

The first of these reactions is important for the Big Bang. The new evaluated cross section takes into account new experimental data for this reaction, and results in a reaction cross section that is about $9 \%$ higher than the previous one [16] at low energies. This implies increased deuterium destruction for Big Bang Nucleosynthesis. The second reaction is one of the most important reactions in nuclear astrophysics - it determines the ${ }^{12} \mathrm{C} /{ }^{16} \mathrm{O}$ ratio in massive stars, and determines whether massive stars will collapse to neutron stars or black holes after supernova explosions. The new evaluated cross section results in an astrophysical S-factor (the cross section with the Coulomb penetrability and geometric cross section divided out) of $S(E 1)=23 \mathrm{keV} \cdot b$, almost a factor of 4 lower than another recent analysis $[17]$. This implies a higher ${ }^{12} \mathrm{C} /{ }^{16} \mathrm{O}$ ratio than is ordinarily used for massive star evolution. Finally, the ${ }^{13} \mathrm{C}(\alpha, n){ }^{16} \mathrm{O}$ reaction is extremely important for the production of neutrons used for the synthesis of elements heavier than $\mathrm{Fe}$ in the slow neutron capture process (s-process). The new evaluated cross section results in a reaction rate that is more than an order of magnitude higher than the previous rate $[18]$ at low temperatures, which makes available significantly more neutrons for the s-process from this reaction than was previously thought.

Recently, a new analysis of low-energy $N+N$ scattering has been started utilizing deuteron photodisintegration data in order to obtain an improved evaluation for 
$n+p$ capture in the region of importance for Big Bang Nucleosynthesis [19]. This reaction contributes large uncertainties in the predicted abundances of primordial deuterium and ${ }^{7} \mathrm{Li}$, and therefore of the Big Bang Nucleosynthesis constraint on the baryon density of the universe - or, equivalently, the amount of normal (baryonic) matter in the universe.

\section{STATISTICAL MODEL CALCULATIONS OF REACTION CROSS SECTIONS}

The calculation of nucleosynthesis and energy generation in supernovae and presupernova stars typically involves thousands of nuclear reactions, many more than can be practically measured, even were the nuclei stable and only in their ground states (neither is true in a supernova). In these situations, calculations of reaction cross sections (which are then converted into rates) are absolutely essential input for astrophysical models. For isotopes with mass $A>24$, the high density of states in the compound nucleus means that most reactions involving the capture or exchange of a single neutron, proton, or $\alpha$-particle (i.e., most of the reactions of astrophysical interest) can be treated using a statistical (Hauser-Feshbach) model.

A lengthy and thorough review of the Hauser-Feshbach cross section calculations employed in modern studies of nucleosynthesis has recently been completed [20]. The study examines the sensitivity of the nucleosynthesis of intermediate mass elements (28<A<80) in supernovae derived from massive stars to the nuclear reaction rates employed in the model. Two standard sources of reaction rate data $[21,22]$ are employed in pairs of calculations that are otherwise identical. Both include as a common backbone the experimental reactions rates of [18]. Two stellar models of 15 and 25 solar masses are evolved from core hydrogen burning to a presupernova state carrying an appropriately large reaction network, then exploded using a piston near the edge of the iron core as described by [23]. The final stellar yields from these models calculated with the two rate sets are compared and found to differ in most cases by less than a factor of two over the entire range of nuclei studied $(1<Z<30$ ). Reasons for the major discrepancies are discussed in detail along with the physics underlying the two reaction rate sets employed. The nucleosynthesis results are relatively robust and less sensitive than might be expected to uncertainties in nuclear reaction rates, though they are sensitive to the stellar model employed.

A new set of reaction rates based on Hauser-Feshbach calculations has been developed by Rauscher and Thielemann [24]. This will be adopted as a new standard by both the Woosley and Theilemann groups and will constitute the source of theoretical reaction rates in future work that will reexamine nucleosynthesis in massive stars [23] and galactic chemical evolution [25]. 


\section{REACTION EVALUATIONS FOR EXPLOSIVE HYDROGEN AND HELIUM BURNING}

Stellar explosions such as novae and X-ray bursters are among the largest thermonuclear explosions known in the cosmos. Sequences of nuclear reactions - primarily proton captures and $(\alpha, p)$ reactions - on stable and on proton-rich radioactive nuclei are thought to power these events. These reactions generate enormous amounts of energy and synthesize isotopes up to mass $A \sim 40$ (novae) and $A \sim 80-100$ (X-ray bursters) [26]. Evaluation of crucial nuclear reactions, especially in the mass $A<20$ and $30<A<50$ ranges, has been designated as a high priority by an ad-hoc Nuclear Astrophysics Data Steering Committee [27].

Evaluations of two important reactions in novae and X-ray bursters - the ${ }^{14} \mathrm{O}(\alpha, \mathrm{p}){ }^{17} \mathrm{~F}$ and ${ }^{17} \mathrm{~F}(\mathrm{p}, \gamma){ }^{18} \mathrm{Ne}$ - have recently been completed. [28] These reactions serve to bypass the slow ${ }^{14} \mathrm{O}$ beta decay, aiter the abundances of oxygen isotopes produced in explosions, and potentially process CNO nuclides up to isotopes with mass $A>20$. The new evaluation corrected an error as large as $13 \%$ in the ${ }^{17} \mathrm{~F}(\mathrm{p}, \gamma){ }^{18} \mathrm{Ne}$ rate, gave the first complete expression for the ${ }^{14} \mathrm{O}(\alpha, \mathrm{p}){ }^{17} \mathrm{~F}$ rate using all current experimental data, determined realistic rate uncertainties, and fit the results using the two most popular rate parameterizations. The evaluations are also posted on the WWW. [29] Another project (in progress) is the evaluation of the ${ }^{18} \mathrm{~F}(\mathrm{p}, \alpha){ }^{15} \mathrm{O}$ and ${ }^{18} \mathrm{~F}(\mathrm{p}, \gamma){ }^{19} \mathrm{Ne}$ reactions. The rates of these reactions help determine the amount of CNO nuclides in stellar material that may be processed up to higher masses [via $(p, \gamma)]$ or cycled back down to lower masses [via $(\mathrm{p}, \alpha)$ ]. The new evaluation is focusing on the contributions of known resonances in ${ }^{19} \mathrm{Ne}$ above the ${ }^{18} \mathrm{~F}+p$ threshold, as well as those of six resonances expected from the level structure of ${ }^{19} \mathrm{~F}$ but never seen in the ${ }^{19} \mathrm{Ne}$ compound system [30]. This evaluation work for explosive hydrogen and helium burning is closely coupled to a measurement program with proton-rich radioactive isotopes at ORNL's Holifield Radioactive Ion Beam Facility [31-33].

Another project vital to improve our understanding of explosive hydrogen burning is the evaluation of $(p, \gamma)$ and $(p, \alpha)$ reactions on isotopes with mass 30-50. Currently, information on level properties, resonance parameters, and cross sections are being compiled for a number of such reactions - for example, ${ }^{31} \mathrm{P}(\mathrm{p}, \gamma),{ }^{31} \mathrm{P}(\mathrm{p}, \alpha)$, ${ }^{32,33,34,36} \mathrm{~S}(\mathrm{p}, \gamma)$, and ${ }^{32,33,34,36} \mathrm{~S}(\mathrm{p}, \alpha)[34]$. The next stage of this project involves organizing the compiled data - summarizing the references, producing tabular data, and posting it on the WWW [35]. Procedures will be developed for calculating the proton capture strengths associated with these $(p, \gamma)$ and $(p, \alpha)$ reactions, and searches will be made for possible missing low-energy proton resonances through consideration of isobaric analog nuclei as well as transfer reaction [e.g., $\left.\left({ }^{3} \mathrm{He}, \mathrm{d}\right)\right]$ data. Depending on their parameters (resonance energy, spin, parity, total and partial widths), such missing resonances can change reaction rates by orders of magnitude. In addition to calculating the reaction rates, procedures have been developed to calculate the associated uncertainties in the reaction rates based on the 
reported experimental uncertainties of the resonance energies, strengths, and other parameters. These uncertainties will also be organized and posted on the WWW.

\section{EVALUATIONS FOR PHYSICS IN RED GIANT STARS}

The relative abundances of the oxygen isotopes are an important diagnostic of the conditions inside red giant stars. The abundance of ${ }^{17} \mathrm{O}$ is particularly sensitive to stellar mixing and convection caused by the extreme temperature dependence of the two nuclear reactions that destroy ${ }^{17} \mathrm{O},{ }^{17} \mathrm{O}(\mathrm{p}, \alpha){ }^{14} \mathrm{~N}$ and ${ }^{17} \mathrm{O}(\mathrm{p}, \gamma)^{18} \mathrm{~F}[36]$. This strong temperature dependence results from the properties of 4 resonances near the ${ }^{17} \mathrm{O}+p$ threshold in ${ }^{18} \mathrm{~F}$. Additionally, measurements of the ${ }^{17} \mathrm{O} /{ }^{16} \mathrm{O}$ and ${ }^{18} \mathrm{O} /{ }^{16} \mathrm{O}$ ratios in pre-solar meteoritic grains have been used to determine the age of the Galaxy [37]. Interpretation of these isotopic ratio measurements is hampered by uncertainties in the rates of the ${ }^{17} \mathrm{O}(\mathrm{p}, \alpha)^{14} \mathrm{~N}$ and ${ }^{17} \mathrm{O}(\mathrm{p}, \gamma){ }^{18} \mathrm{~F}$ nuclear reactions.

In order to better use the oxygen abundances as tracers of red giant convection and of the galactic age, improvements in these two reaction rates are needed. There have been several recent measurements of the properties of these states, and these results are being used in a new evaluation (in progress) of the ${ }^{17} \mathrm{O}(\mathrm{p}, \alpha)^{14} \mathrm{~N}$ and ${ }^{17} \mathrm{O}(\mathrm{p}, \gamma)^{18} \mathrm{~F}$ reactions. Careful consideration is being given to identifying and quantifying the uncertainties in these rates, including contributions from weak resonances and interferences. Preliminary results for this evaluation have motivated a new measurement of the ${ }^{17} \mathrm{O}(\mathrm{p}, \gamma){ }^{18} \mathrm{~F}$ reaction [38] that addresses one of the most significant remaining uncertainties in this rate. The results of this measurement are currently being incorporated in the evaluation.

\section{COMPILATIONS OF CHARGED-PARTICLE INDUCED REACTIONS}

Evaluations of charged particle-induced reactions are crucial in many areas of nuclear astrophysics, and compilations of existing experimental data provide the foundation for all evaluations. Unfortunately, the compilations for charged particleinduced reactions are inadequate, lagging significantly behind those for neutroninduced reactions. The National Nuclear Data Center is addressing this serious problem by compiling charged particle nuclear data in references dating back to 1980 , and entering it into the NNDC databases. Presently there are more than 3,700 such references entered onto the Cross Section Information Storage and Retrieval System (CSISRS) database [39], corresponding to approximately 700,000 data points. The CSISRS database contains a total of over 5 million data points, with information including cross sections, angular and energy distributions, resonance parameters, and polarizations. The format for the data is EXFOR, a worldwide exchange format for experimental nuclear reaction data. 
As part of this project, the NNDC is entering the experimental data used in the NARCRE collection of reaction rates [40] into the NNDC databases, and has entered into a cooperative agreement with VNIIEF (Sarov, Russia) whereby VNIIEF will scan in data from figures in the articles when numerical data is unavailable.

\section{DISSEMINATIONS OF NUCLEAR ASTROPHYSICS DATA}

The projects described in the above sections involve evaluations, calculations, and compilations which expand and improve the nuclear physics information requred for research in astrophysics. However, it is very important to put this information into user-friendly formats that may be easily incorporated into astrophysics models, and to rapidly disseminate this work to the research community.

One example of such dissemination work is the posting of one of the most important reaction rate collections, by Caughlan and Fowler [18], on the WWW. This is the first electronic dissemination [29] of the 160 reaction rates (with inverses) contained in this collection. (Roughly half of these rates have been updated by the NACRE collaboration [40].) The utility of the original Caughlan and Folwer rate collection was improved by making a graphical user interface and search engine based on the chart of the nuclides, by adding GIF and Postscript plots of each of the rates, by developing a technique to automatically regenerate plots to simplify future modifications, by adding a downloadable FORTRAN subroutine of the rates, and by calculating and posting temperature derivatives of the rates. Other sets of reaction rates - for explosive hydrogen burning and for Big Bang nucleosynthesis - are also posted on the WWW, as well as a Nuclear Astrophysics Bibliography [29]. The bibliography is a useful resource for producing evaluations of nuclear reaction and structure information important for astrophysics. It includes references to astrophysical journals and reports which are outside the normal scope of Nuclear Science References [41]. The first phase of this project, which includes over 1200 references, has recently been completed.

Other dissemination projects include the posting on the WWW of reaction rate calculations of Hoffman and Woosley, of Thielemann and collaborators, other rate collections, weak interaction rates, atomic masses, chemical and isotopic abundances, and lists of astrophysics meetings [42].

\section{SUMMARY}

Several recent projects that address the nuclear data needs for astrophysics research have been described. These projects include compilation of charged particle data, evaluations of capture reactions on stable and unstable isotopes, calculations of cross sections with statistical models and R-matrix theory, and dissemination of this information on the WWW. These projects serve to improve the nuclear data 
available for astrophysical studies of important phenomena such as supernovae, novae, $\mathrm{X}$-ray bursts, red giant stars, massive star evolution, and the Big Bang.

\section{ACKNOWLEDGEMENTS}

ORNL is managed by Lockheed Martin Energy Research Corp. for the U.S. Department of Energy under contract number DE-AC05-96OR22464.

\section{REFERENCES}

1. Arnett, D., Supernovae and Nucleosynthesis, Princeton: Princeton Univ. Press, 1996, pp. 424.

2. Timmes, F. X., Woosley, S. E., Hartmann, D. H., Hoffman, R. D., Astrophys. J. 464, 332 (1996).

3. Nagataki, S., Hashimoto, M., Sato, K., Yamada, S., Mochizuki, Y. S., Astrophys. J. Lett. 492, L45 (1998).

4. Mochizuki, Y. S., Kumagai, S., in The Hot Universe: Proc. IAU Symp. No. 188, Kyoto, August 1997, eds. K. Koyama, S. Kitamoto, M. Itoh, Dordrecht: Kluwer Academic, 1998, pp. 241.

5. Diehl, R. et al., Astron. Astrophys. 298, 445 (1995).

6. Knodlseder, J., Astrophys. J. 510, 915 (1999).

7. Timmes, F. X., Woosley, S. E., Hartmann, D. H., Hoffman, R. D., Weaver, T. A., Matteucci, F., Astrophys. J. 449, 204 (1995).

8. Wu, S.-C., and Browne, E., "Table of Radionuclides", LPRI report, Saclay, Feb. 1999.

9. Cameron, J., and Singh, B., Nucl. Data Sheets, $A=44$, June 1999.

10. Wu, S.-C., unpublished evaluation for the Decay Data Evaluation Project, 1999.

11. Fuller, G. M., Fowler, W. A., Newman, M. J., Astrophys. J. Suppl. Ser. 42, 447 (1980); Astrophys. J. Suppl. Ser. 48, 279 (1982); Astrophys. J. 252, 715 (1982).

12. Langanke, K., Martínez-Pinedo, G., Phys. Lett. B453, 187 (1999).

13. Geist, W. H., Brune, C. R., Karwowski, H. J., Ludwig, E. J., Veal, K. D., and Hale, G. M, Phys. Rev. C, in press (1999).

14. Hale, G. M., in Stellar Evolution, Stellar Explosions, and Galactic Chemical Evolution: Proc. 2nd Oak Ridge Symposium on Atomic and Nuclear Astrophysics, Oak Ridge, Dec. 1997, ed. A. Mezzacappa, Bristol: IOP, 1998, pp. 17.

15. Hale, G. M., Chadwick, M. B., Smith, M. S., Bull. Am. Phys. Soc. 44, 262 (1999).

16. Krauss, A., Becker, H. W., Trautvetter, H. P., Rolfs, C., Brand, K., Nucl. Phys. A465, 150 (1987).

17. Ouellet, J. M. L., Butler, M. N., Evans, H. C., Lee, H. W., Leslie, J. R., McArthur, J. D., McLatchie, W., Mak, H.-B., Skensved, P., Whitton, J. L., and Zhao, X., Phys. Rev. C54, 1982 (1996).

18. Caughlan, G. R. and Fowler, W. A., Atomic Data Nuclear Data Tables 40, 283 (1988).

19. Copi, C., Schramm, D. N., Turner, M., Science 267, 192 (1994). 
20. Hoffman, R. D., Woosley, S. E., Weaver, T. A., Rauscher, T., Thielemann, F. -K., Astrophys. J. 521, 735 (1999).

21. Woosley, S. E., Fowler, W. A., Holmes, J. A., and Zimmerman, B. A., Atomic Data Nuclear Data Tables 22, 371 (1978).

22. Thielemann, F. -K., Arnould, M., and Truran, J., in Advances in Nuclear Astrophysics, ed. E. Vangioni-Flam, Gif sur Yvette: Editions Frontière, 1987, pp. 525.

23. Woosley, S. E., Weaver, T. A., Astrophys. J. Suppl Ser. 101, 181 (1995).

24. Rauscher, T. and Thielemann, F.-K., Atomic Data Nuclear Data Tables, in press (1999).

25. Timmes, F. X., Woosley, S. E., Weaver, T. A. Astrophys. J. Suppl Ser. 98, 617 (1995).

26. Wiescher, M., Schatz, H., Champagne, A. E., Phil. Trans. R. Soc. Lond. A 356, 2105 (1998).

27. Parker, P. D. et al., Nuclear Astrophysics Data Steering Committee, unpublished (1996).

28. Bardayan, D. W., Smith, M. S., Phys. Rev. C56, 1647 (1997).

29. http://www.phy.ornl.gov/astrophysics/data/data.html

30. Utku, S., et al., Phys. Rev. C57, 2731 (1998).

31. Smith, M. S., in Origin of Matter and Evolution of Galaxies 97, eds. S. Kubono, T. Kajino, K. I. Nomoto, I. Tanihata, Singapore: World Scientific, 1998, pp. 200.

32. Smith, M. S., Nucl. Inst. Meth. B99, 349 (1995).

33. Bardayan, D. W., et al., Phys. Rev. Lett. 83, 45 (1999).

34. Smith, D. L. and Van Wormer, L. A., in Stellar Evolution, Stellar Explosions, and Galactic Chemical Evolution: Proc. 2nd Oak Ridge Symposium on Atomic and Nuclear Astrophysics, Oak Ridge, Dec. 1997, ed. A. Mezzacappa, Bristol: IOP, 1998, pp. 25.

35. http://isotopes.lbl.gov/isotopes/astro/Reaction-compilation.html

36. El-Eid, M. F., Astron. Astrophys. 285, 915 (1994).

37. Nittler, L. R. and Cowsik, R., Phys. Rev. Lett. 78, 175 (1997).

38. Blackmon, J. C., et al., ORNL Physics Division 1998 Progress Report, http://www.phy.ornl.gov/progress/; Blackmon, J. C., et al., Bull. Amer. Phys. Soc., http://www.aps.org/meet/CENT99/BAPS/abs/S3415002.html.

39. http://www.nndc.bnl.gov/nndc/exfor/

40. http://pntpm.ulb.ac.be/nacre.htm

41. http://www.nndc.bnl.gov/nndc/nsr/nsrframe.html

42. http://ie.lbl.gov/astro.html 